\title{
Keynote Lecture 3
}

\section{S17}

\section{The Integration of Innovative Biomedical and Psychosocial Strategies into Clinical Practice}

\begin{abstract}
Ian R. H. Falloon
Advanced Research Institute for Effective Treatment and Education: ARIETE, Perugia, Italy

In recent years advances in neurobehavioural research have begun to contribute to the development of more effective therapeutic interventions. It is now evident that the vulnerability to schizophrenic disorders is complex, and includes combinations of factors such as genetics, brain injury, endogenous and exogenous toxins. However, as with most major illnesses, the biomedical factors are only part of the equation, and psychosocial stressors appear to play an important role in determining the onset and long-term course of the disorder. Comprehensive treatment that integrates the strategies designed to modify the biomedical factors with those designed to modify the social and psychological factors appears to lead to substantial improvements in the rates of recovery from the impairment, disability and handicaps associated with these disorders. The application of these integrated treatment strategies requires a teamwork approach that ensures that optimal treatment is individualized, targeted to recovery of functioning, and is provided throughout the entire course of the disorder. Such an approach has been demonstrated as feasible in centers throughout the world. However, only a tiny proportion of cases are receiving the benefits of such an approach. The challenge for the new century is to ensure that every person with a schizophrenic disorder receives the optimal treatment until they have all made a full and lasting recovery.
\end{abstract}

